\title{
Combine effect of 5-azacytidine and tgf- $\beta$ in differentiation of mesenchymal stem cells towards cardiomyocytes
}

\begin{abstract}
Myocardial infarction (MI) is a leading cause of morbidity and mortality worldwide. Although pharmaceutical therapy and revascularization strategies are able to delay ventricular remodeling, until today no therapeutic strategy is available that might prevent or reverse the process of remodeling and ventricular failure. In the recent past stem cells regenerative therapy has been presented the best tool for treatment of MI. Bone marrow derived mesenchymal stem cells (MSCs) can be differentiated into different types of cells. In the current study, bone marrow derived MSCs were isolated from femur and tibia of Balb $\mathrm{C}$ Mice and were cultured in 5-azacytidine and $10 \mathrm{ng} / \mu 1$ transforming growth factor (TGF- $\beta$ ) in different groups. The RT-PCR analysis demonstrated that combine treatment of MSCs with 5-azacytidine and TGF- $\beta$ have high effect on MScs differentiation towards cardiomyocytes as compare to 5 -azacytidine and TGF- $\beta$ alone. The cardiac specific genes GATA 4 and cTnT were highly expressed in the combined 5-azacytidine+TGF- $\beta$ treated cells, while GAPDH was used as control. The present study is anticipated to put forward valued aspect of differentiation of bone marrow derived MSCs into cardiac cells. This might assist as a means for improved cellular treatment and might enhance the possibility of improved myocardial regeneration.
\end{abstract}

Keywords: myocardial infarction, mscs, tgf- $\beta$, gapdh, ace, dnmts
Volume 3 Issue I - 2017

\author{
Sulaiman Shams, Muhammad Naseer, \\ Hammad Hassan \\ Department of Biochemistry, Abdul Wali Khan University, \\ Pakistan
}

\section{Correspondence: Sulaiman Shams, Department of} Biochemistry, Stem Cell Regenerative Medicine Lab, Abdul Wali Khan University, Mardan-23200, Khyber Pakhtunkhwa, Pakistan Tel+923339202865, Email Sulaiman@awkum.edu.pk

Received: May 25, 2017| Published: July 10, 2017

\section{Introduction}

Stem cells play an important role in biological research nowadays. It is a vast field holding a great potential in therapeutic approach. The purpose of the stem cell therapy is to treat injured tissues by preventing the dead cells. ${ }^{1}$ Stem cells are undifferentiated having the ability of self-renewal providing these cells in life throughout. ${ }^{2}$ Mesenchymal stem Cells (MSCs) are multipotent stem cells that reside in stromal part of bone marrow and hold a vast clinical application. They have the ability of self-renewal and can be differentiated into a variety of cells. Mesenchymal stem cells differentiated into cardiomyocytes, osteocytes, adipocytes and chondrocytes. ${ }^{3}$ In cardiac regeneration, mesenchymal stem cells have the capability to distinguish towards cardiomyocytes both in vitro and in vivo. ${ }^{4}$

Myocardial infarction commonly and coronary disease are the two severe cardiovascular disorders. South Asia is in great risk of these two cardiovascular diseases than other continents of the world. ${ }^{5}$ Instead of latest therapeutic strategies of heart failure including inhibition of neural hormone with beta blockers and angiotensin-converting enzyme (ACE) inhibitors that satisfy clinical outcome, other methods like interventional therapeutics don't completely end ventricular remoulding because they don't replace injured cells of myocardium. The mortality and morbidity associated with myocardial infarction, novel methods are needed to improve the function of cardiac. ${ }^{6}$ After myocardial infarction, there is no ability of cardiac muscles to redevelop. The death of myocardial cells is brought by ischemia, due to which alerting and decreased contractibility of cardiac takes place. Repairing of the damage cells take place by two approaches. First by replication of endogenous heart cells and secondly by conversion of stem cells into cardiomyocytes. ${ }^{6}$ Myocardial infarction can be treated by regenerative medicines. The plasticity of myocardial cells is done by the replacement of scare tissues with active myocardial cells that enhances neo-angiogenesis. The latest experimental and preclinical studies have given inspiring effects. Bone marrow derived mesenchymal stem cells play a vital role in cardio plasticity. These Bone marrow derived mesenchymal stem cells are the best means of repairing of infarcted cells. ${ }^{7}$ Currently there are two methods to convert bone marrow mesenchymal stem cells into cardiomyocytes in vitro, first by induction and second by co-culturing with cardiomyocytes. ${ }^{8}$ The infarcted myocardial cells replaced with mesenchymal stem cells improve the cardiac function by the production of new myocardial cells and cytokines acting as fibroblast angio-vascular endothelial growth factors which proves that that MSCs enhances the cardiac function by paracrine mechanism and stimulate angiogenesis in the infarcted region of heart. ${ }^{9}$

DNA methylation and histone deacetylation have been revealed to play significant role in stem cell differentiation. Treatment of bone marrow derived stem cells with 5-azacytidine (5-Aza) led to the variations in stem cells with fibroblast-like morphology into freely beating cardiomyocytes. DNA methylation seems to be facilitated by a group of enzymes called deoxyribonucleic acid methyltransferases (DNMTs) that covalently bond a methyl functional group to the cytosine residue inside 5-CpG-3 prime islands at the promoter region. After DNA methylation, a separate group of proteins having a methyl cytosine binding dominion (MBD) is take on and bound to these methylated $\mathrm{CpG}$ sites, which then block the entrance of transcription factors that naturally bind to the promoter. The finale result is a shortened chromatin that further reduces the entrance of 
transcription features to their promoter required sites, finally resulting in protein sequence silencing. ${ }^{10}$ Currently 5 -azacytidine is proficient of bringing the distinction of stem cells into different types of cells. It brings changes in gene expression and transcription in cell that lead to apoptosis or cellular differentiation. 5-Azacytidine is very effective demethylating agent in cardiomyocytes research. ${ }^{11}$ Treatment of bone marrow mesenchymal stem cells with 5-azacytidine results in the formation of cardiac like cells. ${ }^{12}$ Demethylating agents have grown interest because of their epigenetic reprogramming ability in stem cell differentiation. Treatment of stem cells with 5-azacytidine which is a DNA demethylating agent can initiate differentiation of bone marrow derived mesenchymal stem cells into cardiac muscle-fibroblast like cells. $^{13} 5$-azacytidine is potent inhibitor of DNA methylation and has been used as demethylating agents in vitro. In the absence of 5azacytidine results in the activation of normally silenced genes and contribute to tumourigenesis, poses as a major obstacle to epigenetic therapy. ${ }^{14}$ 5-azacytidine (5-aza), a DNA demethylating chemical compound, brings BMMSCs to distinguish into cardiomyocytes by random demethylation. ${ }^{15}$

Bone marrow derived MSCs can also be differentiated into cardiomyocytes by transforming growth factor-beta (TGF- $\beta$ ). ${ }^{16}$ TGF- $\beta$ is a protein which works in increase, differentiation, and some other roles in many types of cells. Numerous cells produce TGF- $\beta$ that controls the activities of many growth factors. They have the ability to improve formation of connective tissue in regeneration. ${ }^{17}$ The TGF$\beta$ is family that comprises of a huge family of cytokines regulating a mass of cellular activities and disease pathogenesis. The family is distributed into three main catagories TGF- $\beta$, nodal branches and GDF. These growth factors reactions are reliant on cellular situation comparatively because of Smad connections with cell kind of specific transcription aspects. TGF- $\beta$ plays key roles in the regulation of normal cell development. ${ }^{18}$ Transforming growth factor-beta (TGF- $\beta$ ) is play an important role in cardiac damage repair and cell proliferation. TGF- $\beta$ is cytokine and is associated in a range of cell purposes such as in the regulating of inflammation, differentiation, growth and extracellular matrix deposition. ${ }^{19}$ The aim of the study was to discover the differentiation potential of bone marrow derived mesenchymal stem cells towards cardiomyocytes by treating bone marrow derived mesenchymal stem cells with 5-azacytidine and TGF- $\beta$.

\section{Material and methods}

Male Albino mice (Balb C) of about 20-25gm, age 6-8weeks, were obtained from National institute of health Islamabad, Pakistan. The animals were kept in a specific pathogen-free room and fed a standard chow diet. All the procedures were carried out according to the guideline for use and care of laboratory animals and approved from bioethics committee of biochemistry department AWKUM.

\section{Sacrificing the mice}

Balb C Mice (20-25gm) were sacrificed after anesthesia with chloroform. Femur and tibia were separated from the body, separate from adherent soft tissue and then washed with phosphate buffer saline (PBS, GIBCO). The two bones were then placed in the petri plate containing PBS.

\section{Isolation of bone marrow derived mesenchymal stem cells}

Bone marrow was collected from tibia and femur with help of disposable $1 \mathrm{ml} \mathrm{DB}$ syringe by flushing the bones with medium (DMEM, GIBCO) supplemented with 10\% Fetal Bovine serum (FBS) of company (BIOWEST), 100units $/ \mathrm{ml}$ penicillin and $100 \mu \mathrm{g} / \mathrm{ml}$ streptomycin (CAPRICON). Approximately $1 \times 10^{6}$ cells were cultured in $25 \mathrm{~cm}^{2}$ culture flask. The culture flasks were incubated at $37^{\circ} \mathrm{C}$ with $5 \% \mathrm{CO}_{2}$. The non-adherent hemapoietic cells were removed from adherent mesenchymal stem cells by removing the culture medium on day 3 . The medium was changed after 3 days. The culture flask was then kept in $\mathrm{CO}_{2}$ incubator containing humid environment at $37^{\circ} \mathrm{C}$. The medium was changed after every three days. The cells were observed under inverted phase contrast microscope (Leica).

\section{Treatment of MSCs with 5-Azacytidine}

MSCs were subculture with $0.25 \%$ trypsin EDTA upto second passage. At passage three cell were cultured in gelatin coated 24 wells plated and working solution of 5-azacytidine (abcam) was added to the working (DMEM) media which was then added to MSCs of group 2. The cells were again incubated in $5 \% \mathrm{CO}_{2}$ incubator at $37^{\circ} \mathrm{C}$. The cells were then washed twice with phosphate buffer saline (PBS, GIBCO) and the medium was replaced with normal DMEM. The experiments were terminated after 15 days.

\section{Treatment of MSCs with mTGF- $\beta$}

The working 10ng/ul TGF- $\beta$ (Bio Vision) solution was transferred to the working Dulbecco's modified Eagle's medium (DMEM) and was then added to the culture cells of group 3 containing MSCs. The cells were incubated in $5 \% \mathrm{CO}_{2}$ incubator at $37^{\circ} \mathrm{C}$. The cells were then washed twice with phosphate buffer saline (PBS) and the medium was changed with normal DMEM. The experiments were terminated after 15days.

\section{Combined treatment of BM-MSCs with 5-azacytidine \& TGF}

Solutions of 5-azacytidine and TGF- $\beta$ were both added to the working Dulbecco's modified Eagle's medium (DMEM) and was then added to the culture cells of group 3 containing MSCs. The cells were incubated in $5 \% \mathrm{CO}_{2}$ incubator at $37^{\circ} \mathrm{C}$. The cells were then rinsed twice with phosphate buffered saline and the medium was changed with normal working medium. The experiments were completed after 15days.

\section{Primers for the study}

The primers used for the study of differentiation of stem cells were synthesized by Molecular Biology Product Karachi, Pakistan. GAPDH was used as internal control. The expression level of cardiomyocytes specific markers i.e. cTnT and GATA4 were also checked in treated and untreated stem cells. Their product size and sequence are given in the following table (Table 1)

\section{RNA Isolation from cells and cDNA synthesis}

RNA was isolated from normal and treated cells by using Trizol reagent. cDNA was synthesis using Invitrogen kit. The cDNA was stored at $-20^{\circ} \mathrm{C}$ for further analysis for Polymerase chain reaction.

\section{Amplification of cDNA and gel documentation}

For PCR analysis $1 \mu \mathrm{L}$ of cDNA was taken and amplified using thermal cycler (ABI). 35 cycles of PCR amplification were performed according to the kit (INVITROGEN). The sample was run on $1.5 \%$ 
agarose gel. 100bp DNA ladder (INVITROGEN) was load in the first well of the gel and run the gel at 120 Volts for 50minutes. The DNA fragments were visualized under UV light with the help of gel documentation system.

Table I List of primers with their sequences and product size

\begin{tabular}{|c|c|c|c|}
\hline $\begin{array}{l}\text { PCR } \\
\text { primer }\end{array}$ & Sequence & $\begin{array}{l}\text { Annealing } \\
\text { temp }\end{array}$ & $\begin{array}{l}\text { Size } \\
\text { in bp }\end{array}$ \\
\hline $\begin{array}{l}\mathrm{cTnT}(\mathrm{F}) \\
\mathrm{cTnT}(\mathrm{R})\end{array}$ & $\begin{array}{l}\text { TTCGACCTGCAGGAAAGTT } \\
\text { GTGCCTGCAAGACCTAGAG }\end{array}$ & $60^{\circ} \mathrm{C}$ & 206 \\
\hline $\begin{array}{l}\text { GATA4 (F) } \\
\text { GATA4 (R) }\end{array}$ & $\begin{array}{l}\text { TCTCACTATGGGCACAGCAG } \\
\text { CCGAGCAGGAATTTGAAGAG }\end{array}$ & $60^{\circ} \mathrm{C}$ & 245 \\
\hline $\begin{array}{l}\text { GAPDH (F) } \\
\text { GAPDH (R) }\end{array}$ & $\begin{array}{l}\text { GGAAAGCTGTGGCGTGATGG } \\
\text { GTAGGCCATGAGGTCCACCA }\end{array}$ & $57^{\circ} \mathrm{C}$ & 414 \\
\hline
\end{tabular}

\section{Results}

\section{Isolation of bone marrow derived MSCs}

Albino mice (Balb C) was sacrificed by anesthesia with chloroform and mesenchymal stem cells were isolated from the bone marrow of tibia and femur. The cells were grown in DMEM media supplemented with $10 \%$ FBS and penicillin/streptomycin. The adherent mesenchymal stem cells were observed under inverted phase contrast microscope (Figure 1).

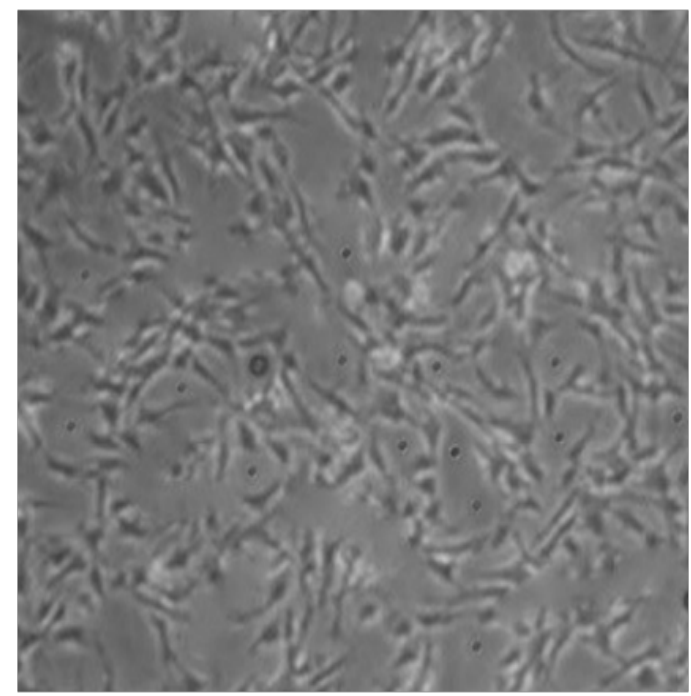

Figure I Morphology of MSCs at passage 3.

\section{Expression of genes in treated cells by RT-PCR}

The expression of cardiac specific genes GATA4 and cTnT were studied at mRNA level by RT-PCR. GATA4 and cTnT cardiac specific genes were expressed in all treated cells. The GATA4 and cTnT was highly expressed in combined treated cells (aza+mTGF- $\beta$ ) as shown in group 3 . The cardiac specific markers were not expressed in untreated cells (Figure 2A \& 2B). GAPDH was used as control as shown in Figure 2C.

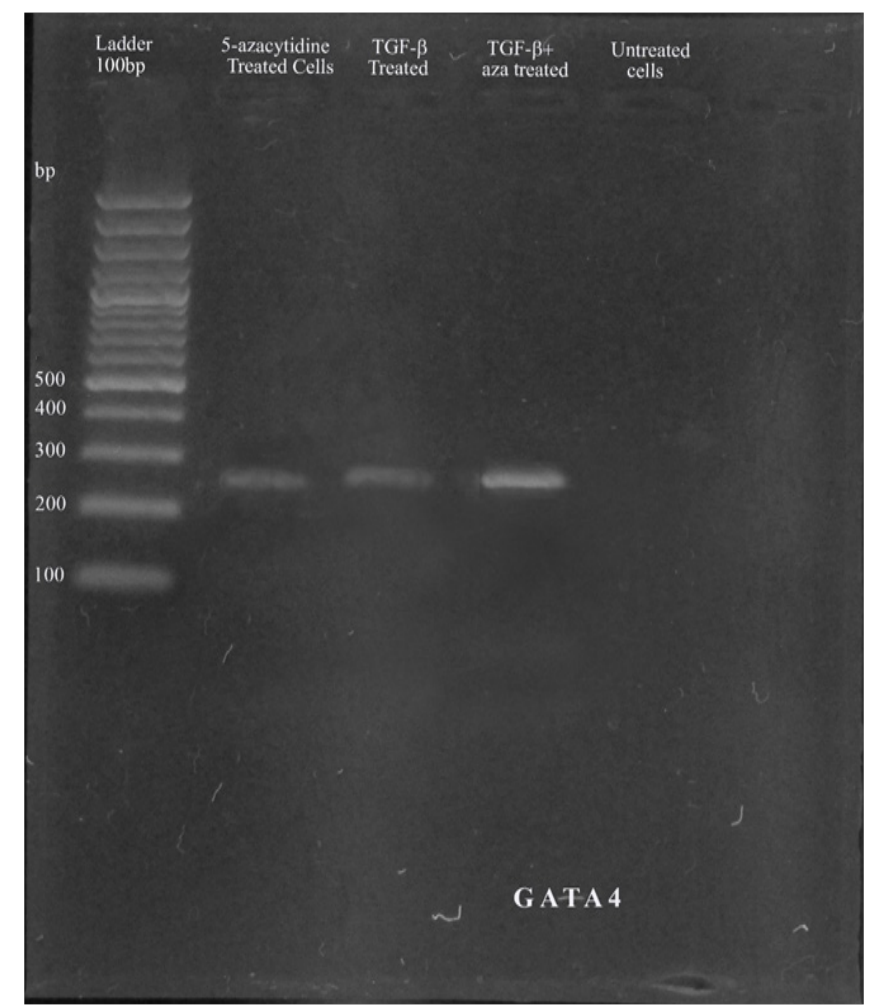

Figure 2A Expression of GATA4, line I: I 00bp Ladder, line 2: aza-treated cells, line 3:TGF- $\beta$ treated cells, line 4: combined treated cells, line 5: untreated cells.

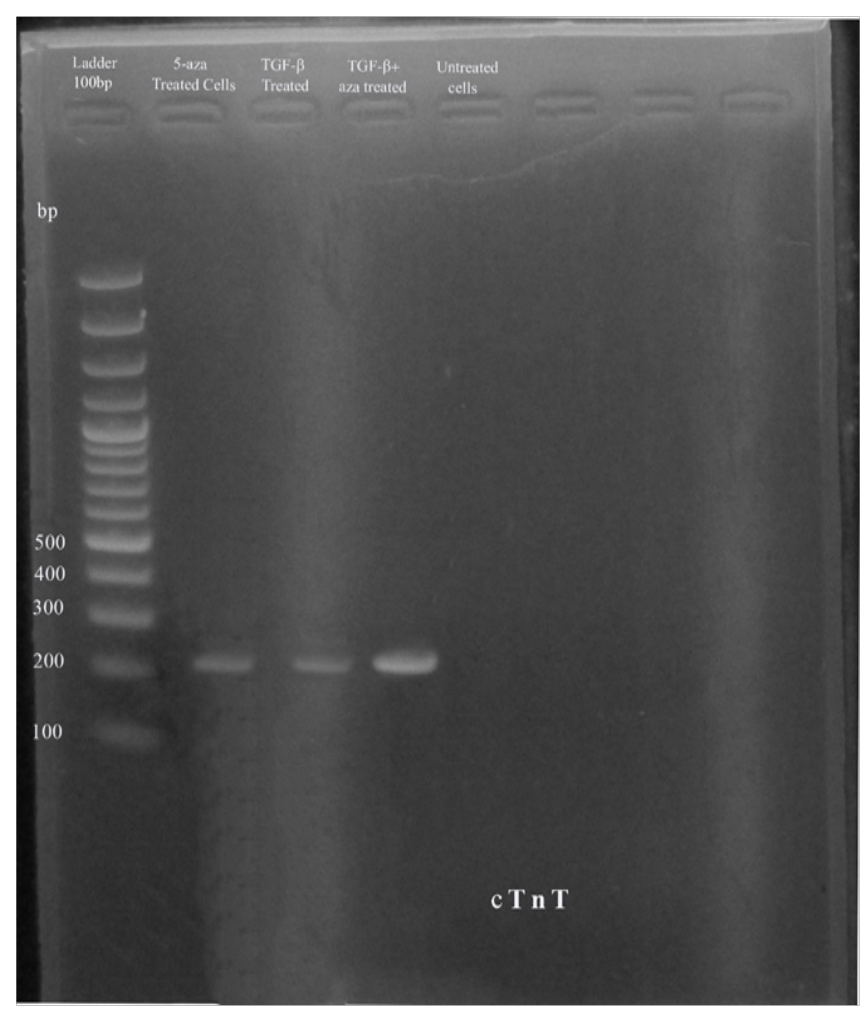

Figure 2B Expression of cTnT, line I: I00bp Ladder, line 2: aza-treated cells, line 3:TGF- $\beta$ treated cells, line 4: combined treated cells, line 5 : untreated cells. 


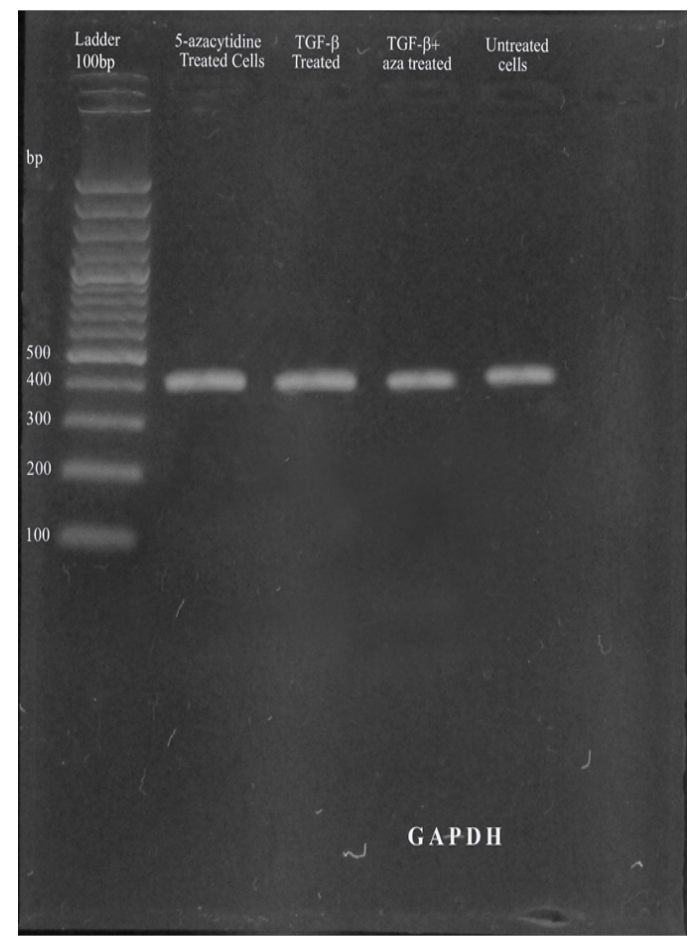

Figure 2C Expression of GAPDH, line I: I00bp Ladder, line 2: aza-treated cells, line 3:TGF- $\beta$ treated cells, line 4: combined treated cells, line 5 : untreated cells.

\section{Discussion}

Myocardial infarction is the permanent death of heart cells secondary to persistent lack of oxygen in the body. Myocardial infarction is generally caused by blood coagulate, that breaks the blood movement to the part of cardiac cells. ${ }^{20}$ Treatment of myocardial infarction with clot-breaking medicine procedure is usually done to bring back the blood flow over the clogged blood vessel are commonly done. ${ }^{21}$ The injured tissues of the heart in myocardial infarction can also be repaired by the replacement of damaged cells using stem cell therapy in cellular transplantation. Bone marrow derived mesenchymal stem cells are multipotent stem cells that are the main source of cell therapy to repair injured tissues of myocardial infarction. Some in vitro experiments have shown that very limited section of MSCs changed phenotypically into cardiomyocytes that has affected their use in myocardial treatment. The reason behind it may be the ability of these cells to differentiate into different types of cells resulting in very limited amount of these cells are induced into cardiomyocytes. Therefore, a method needs to be developed to enhance the differentiation ability of these stem cells into cardiomyocytes, so that MSCs can be differentiated into cardiac specific cells up to some extent by directing them before transplantation. Such method can increase their efficiency for cardiac therapy. ${ }^{22}$

The differentiation ability of these cells is controlled by the use of epigenetic changes which increases their differentiation ability. The two complementary events i.e. methylation and demethylation are responsible for epigenetic changes that take place in DNA or in protein modification. ${ }^{23}$ Gene expression is regulated by methylation. There is a close relationship between methylation and gene expression. Methylation of some proteins is carried out in the presence of an enzyme called histone methyl transferases that causes DNA binding proteins (histones) at the region of lysine site. Differentiation and selfrenewal ability are maintained by methylation and its complementary process demethylation. ${ }^{24}$

Methylation works in conservation of undifferentiated state of MSCs regulator and demethylation works in differentiation process of these MSCs. ${ }^{25}$ Cardiogenic differentiation in MSCs can be induced by 5 -azacytidine and cardiac specific growth factor such as mTGF- $\beta$. 5 -azacytidine acts as demethylating agent and express cardiomyocytes specific genes. 5-azacytidine can be fused into DNA of specific genes to carry out covalent bond with an enzyme DNA methyltransferase. It results in reduction in enzyme action that results demethylation of DNA of specific gene. ${ }^{26}$

It is accepted that demethylation began by 5-azacytidine enhance the expression of many genes. In the current study it was observed that cardiac specific genes (TNT, GATA4) were highly expressed in all treated bone marrow derived MSCs. These pro play a significant Part in the manner of induced differentiation. Bone marrow derived MSCs treated with 5-azacytidine resulted in differentiation of bone marrow derived MSCs into cardiomyocytes by expressing cardiac specific genes. Thus they have the ability to replace injured cardiac cells in heart attack. ${ }^{27}$

TGF- $\beta$ signaling is vital for suppression of seditious gene synthesis in curing infarcted cells. The cardiac specific growth factor TGF- $\beta$ may play an important role in modulating fibroblast phenotype and gene expression, helping extracellular matrix removal in the infarcted cells by increasing collagen and fibronectin synthesis and by decreasing matrix deprivation through introduction of enzyme called protease inhibitors. ${ }^{28}$ In present study bone barrow derived MSCs treated $10 \mathrm{ng} / \mathrm{ml}$ with TGF- $\beta$ for 15 days also resulted in differentiation of MSCs into cardiomyocytes by the expression of cardiac specific genes. Balb C Mice bone marrow derived MSCs were treated with both 5 -azacytidine and TGF- $\beta$ in present study. The alteration in mRNA levels of Cardiac specific genes in treated MSCs were studied by RT-PCR. The gene expression by RT-PCR indicated that treated MSCs have expressed cardiac-specific genes such as GATA4 and cTnT, showing that the treated MSCs had gotten cardiomyogenic genotype while combined treated cells (azcytidine + mTGF- $\beta$ ) were highly expressed as shown in Figure 2A \& 2B indicating that the cells were highly differentiated.

Thus it was concluded that combination treatment of MSCs with 5 -azacytidine and TGF- $\beta$ might be inducing normal regeneration of abnormal myocardial tissues after myocardial infarction. Therefore, transplantation of these cells can become a better option for bringing normal myocardial function. The treatment of these multipotent cells with 5 -azacytidine and TGF- $\beta$ can express cardiac specific genes and these cells holds the power of differentiation towards cardiomyocytes. Due to this reason 5 -azacytidine and TGF- $\beta$ treated bone marrow derived MSCs are reflected as the hopeful source of stem cell therapy in myocardial infarction.

\section{Conclusion}

The current study determined that bone marrow derived MSCs treated with 5 -azacytidine and TGF- $\beta$ can differentiate towards cardiomyocytes. The 5 -azacytidine and TGF- $\beta$ treated cells expressed the cardiac specific genes. The combined treated cells were highly expressed for cardiac specific genes. Therefore, it is concluded that 
5-azacytidine and TGF- $\beta$ treated bone marrow derived MSCs can enhance the probabilities of successful regeneration of injured cardiac cells before transplantation and their combined treatment can enhance the differentiation potential.

\section{Acknowledgements}

We are highly thankful to Directorate of Science \& Technology (DoST) Khyber Pakhtunkhwa, Pakistan for financial support.

\section{Conflict of interest}

The author declares no conflict of interest.

\section{References}

1. Orlic D, Kajstura J, Chimenti S, et al. Bone marrow stem cells regenerate infarcted myocardium. Pediatr transplant. 2003;7(Suppl 3):86-88.

2. Muller-Sieburg C, Sieburg HB. Stem cell aging:survival of the laziest? Cell Cycle. 2008;7(24):3798-3804.

3. Minguell JJ, Erices A. Mesenchymal stem cells and the treatment of cardiac disease. Exp Biol Med. 2006;231(1):39-49.

4. Jackson L, Jones DR, Scotting P, et al. Adult mesenchymal stem cells:differentiation potential and therapeutic applications. J Postgrad Med. 2007;53(2):121.

5. Saleheen D, Zaidi M, Rasheed A, et al. The Pakistan Risk of Myocardial Infarction Study: a resource for the study of genetic, lifestyle and other determinants of myocardial infarction in South Asia. Eur J Epidemiol. 2009;24(6):329-338.

6. Kumar R, Sharma A, Pattnaik AK, et al. Stem cells: An overview with respect to cardiovascular and renal disease. J Nat Sci Biol Med. 2010;1(1):43-52.

7. Akar AR, Durdu S, Cubukçuoğlu Deniz G, et al. Failing heart; remodel, replace or repair? Anadolu Kardiyol Derg. 2008;8(Suppl 2):148-157.

8. Beier JP, Bitto FF, Lange C, et al. Myogenic differentiation of mesenchymal stem cells co-cultured with primary myoblasts. Cell Biol Int. 2011;35(4):397-406.

9. Huang NF, Lee RJ, Li S. Chemical and physical regulation of stem cells and progenitor cells:potential for cardiovascular tissue engineering. Tissue engineering. 2007;13(8):1809-1823.

10. Krishnan M, Park JM, Cao F, et al. Effects of epigenetic modulation on reporter gene expression:implications for stem cell imaging. FASEB $J$. 2006;20(1):106-108.

11. Lavelle D, Saunthararajah Y, Desimone J. DNA methylation and mechanism of action of 5-azacytidine. Blood. 2008;111(4):2485-2485.

12. Grajales L, García J, Banach K, et al. Delayed enrichment of mesenchymal cells promotes cardiac lineage and calcium transient development. $J$ Mol Cell Cardiol. 2010;48(4):735-745.
13. Naeem N, Haneef K, Kabir N, et al. DNA Methylation Inhibitors, 5azacytidine and Zebularine Potentiate the Transdifferentiation of Rat Bone Marrow Mesenchymal Stem Cells into Cardiomyocytes. Cardiovasc Ther. 2013;31(4):201-209.

14. Yoo CB, Cheng JC, Jones PA. Zebularine: a new drug for epigenetic therapy. Biochem Soc Trans. 2004;32(Pt 6):910-912.

15. Xing Y, Lv A, Wang L, et al. The combination of angiotensin II and 5azacytidine promotes cardiomyocyte differentiation of rat bone marrow mesenchymal stem cells. Mol Cell Biochem. 2012;360(1-2):279-287.

16. Lefer AM, Tsao P, Aoki N, et al. Mediation of cardio protection by transforming growth factor-beta. Science. 1990;249(4964):61-64.

17. Sporn MB, Roberts AB, Wakefield LM, et al. Transforming growth factor-beta:biological function and chemical structure. Science. 1986;233(4763):532-534

18. Harish RC, Abidali M, David IC, et al. Transforming growth factor-b, MAPK and Wnt signaling interactions in colorectal cancer. EuPA Open Proteomics. 2015;8:104-115.

19. Rath D, Chatterjee M, Müller I, et al. Platelet expression of transforming growth factor beta 1 is enhanced and associated with cardiovascular prognosis in patients with acute coronary syndrome. Atherosclerosis. 2014;237(2):754-759.

20. Konstantinidis K1, Whelan RS, Kitsis RN. Mechanisms of cell death in heart disease. Arterioscler Thromb Vasc Biol. 2012;32(7):1552-1562.

21. Goldhaber SZ, Grasso-Correnti N. Treatment of blood clots. Circulation. 2002;106:e138-e140.

22. Mummery CL, Zhang J, Ng ES, et al. Differentiation of human embryonic stem cells and induced pluripotent stem cells to cardiomyocytes a methods overview. Circulation research. 2012;111(3):344-358.

23. Jin B, Li Y, Robertson KD. DNA Methylation Superior or Subordinate in the Epigenetic Hierarchy? Genes Cancer. 2011;2(6):607-617.

24. Kosan C, Godmann M. Genetic and Epigenetic Mechanisms That Maintain Hematopoietic Stem Cell Function. Stem Cells Int. 2016;2016:5178965.

25. Farshdousti Hagh M, Noruzinia M, Mortazavi Y, et al. Different methylation patterns of RUNX2, OSX, DLX5 and BSP in osteoblastic differentiation of mesenchymal stem cells. Cell J. 2015;17(1):71-82.

26. Qian Q, Qian H, Zhang X, et al. 5-Azacytidine induces cardiac differentiation of human umbilical cord-derived mesenchymal stem cells by activating extracellular regulated kinase. Stem cells Dev. 2012;21(1):6775.

27. Pittenger MF, Martin BJ. Mesenchymal stem cells and their potential as cardiac therapeutics. Circ Res. 2004;95(1):9-20.

28. Streuli CH, Schmidhauser C, Kobrin M, et al. Extracellular matrix regulates expression of the TGF-beta 1 gene. J Cell Biol. 1993;120(1):253260 